\title{
Combined Protocols for Corneal Collagen Cross- Linking with Photorefractive Surgery for Refractive Management of Keratoconus: Update on Techniques and Review of Literature
}

\author{
Angela Y. Zhu $\cdot$ Albert S. Jun · Uri S. Soiberman
}

Received: May 15, 2019

(C) The Author(s) 2019

\begin{abstract}
With the development and gradual dissemination of corneal collagen cross-linking (CXL) in the twenty-first century as an early treatment for keratoconus, the management paradigm has shifted to include a greater focus on complete refractive correction for these patients. Though supplemental hard contact lens therapy remains a mainstay of visual rehabilitation in keratoconus, there has been increasing appeal in a completely surgical approach by combining CXL with adjuvant refractive procedures to both halt the ectatic process and enhance functional visual outcomes. Collectively termed "CXL plus" procedures, several combined protocols have been studied to various degrees in conjunction with CXL, involving photorefractive keratectomy (PRK), transepithelial phototherapeutic keratectomy (PTK), conductive keratoplasty (CK), intrastromal corneal ring segments (ICRS) implantation, phakic intraocular lens (PIOL) implantation, or multiple of these techniques together. The scope of this review aims to encompass a summary of current
\end{abstract}

Enhanced Digital Features To view enhanced digital features for this article go to https://doi.org/10.6084/ m9.figshare.9746588.

A. Y. Zhu · A. S. Jun · U. S. Soiberman ( $\square)$

Wilmer Eye Institute, Johns Hopkins Medical

Institutions, Baltimore, MD, USA

e-mail: usoiber1@jhmi.edu
CXL protocols and present the current status of studies involving adjunctive keratorefractive procedures combined with CXL. By discussing the results to date of these CXL plus protocols, we can assess what further areas of investigation are necessary within this field as the next step to optimizing treatment modalities and outcomes for our keratoconus patients, regardless of disease severity.

Keywords: Combined CXL protocol; Corneal collagen cross-linking (CXL); CXL plus; Keratoconus; Photorefractive surgery

\section{INTRODUCTION}

With the advent of corneal collagen cross-linking (CXL) in 1997 and its gradual acceptance in the beginning of the twenty-first century, the treatment paradigm for keratoconus and other corneal ectatic disorders has shifted dramatically [1]. In general, visual rehabilitation in keratoconus involves both managing the ectatic process and addressing the myopic and astigmatic refractive changes resulting from the abnormal corneal architecture. The latter was formerly accomplished by conservative management with hard contact lens correction until disease progression was too significant, requiring corneal transplantation to obtain a more normalized corneal surface. In the two decades 
since CXL was introduced, multiple prospective studies have firmly established its role as an integral aspect of the management of early keratoconus to tackle the previously unaddressed component of halting the ectatic process [2]. However, CXL alone in advanced but progressive disease does not lead to visual rehabilitation, so these patients still rely on contact lens correction or other forms of treatment, which will be discussed herein.

Keratoconus is a bilateral corneal disease characterized by progressive but self-limited thinning and associated steepening of the central or paracentral cornea [3]. Reported prevalence rates range from 50 to 265 per 100,000 , depending on a variety of environmental and genetic factors, but it remains the primary indication for corneal transplantation throughout most of the world (specifically, in Europe, Australia, the Middle East, Africa, and South America) [4-7]. While the pathophysiology of the disease remains elusive, alterations in stromal matrix collagen production and apoptosis of anterior stromal keratocytes are hallmarks of the disorder and trigger pathologic changes in corneal shape and biomechanics [8-11]. Given the likely polygenetic nature of keratoconus and multifactorial contribution of associated conditions (e.g. atopy), it has been difficult to develop an etiology-based cure for prevention of disease progression [12].

CXL is a minimally invasive procedure utilizing riboflavin and ultraviolet-A (UVA) light to increase the biomechanical stability of the cornea and halt ectatic progression by inducing intrastromal cross-links [13]. While CXL has been shown to improve corneal curvature and maintain best-corrected visual acuity (BCVA) over long-term follow-up periods, most patients with moderate to advanced disease still need supplemental refractive correction to achieve functional vision [14-16]. For this reason, there has been increasing appeal in combining CXL with adjuvant refractive procedures to provide both disease stability and enhanced functional visual correction. Coined "CXL plus" in 2011, there are now several combined protocols involving photorefractive keratectomy (PRK), transepithelial phototherapeutic keratectomy (PTK), conductive keratoplasty (CK), intrastromal corneal ring segment (ICRS) implantation, phakic intraocular lens (PIOL) implantation, or multiple of these techniques in conjunction with CXL. This review aims to summarize the current status of studies surrounding these combined CXL plus techniques and discuss the future potential of these protocols.

\section{Compliance with Ethics Guidelines}

This article is based on previously conducted studies and does not contain any studies with human participants or animals performed by any of the authors.

\section{CORNEAL COLLAGEN CROSS- LINKING (CXL) PROTOCOLS}

\section{Conventional (Dresden) Protocol}

The original CXL protocol developed by Wollensak et al. has been termed the conventional or Dresden protocol [13]. This involves debridement of the central $8-9 \mathrm{~mm}$ zone of corneal epithelium after application of topical anesthesia in a sterile setting, then instilling a solution of $0.1 \%$ riboflavin in $20 \%$ dextran every $2 \mathrm{~min}$ for $30 \mathrm{~min}$. After this pre-treatment phase, UVA light $(370 \mathrm{~nm})$ is used to irradiate the cornea for $30 \mathrm{~min}$ at $3 \mathrm{~mW} / \mathrm{cm}^{2}$ irradiance $\left(5.4 \mathrm{~J} / \mathrm{cm}^{2}\right.$ total energy), during which the riboflavin solution is again administered every 2 min. With a minimum central corneal thickness of $400 \mu \mathrm{m}$ prior to UV irradiation, no collateral damage to the endothelium or other intraocular structures is expected $[17,18]$.

Studies on conventional CXL show that the procedure is highly effective, with a mean reduction in maximum keratometry readings of $1.6 \pm 4.2 \mathrm{D}$ at 12 months postoperatively in a US multicenter clinical trial, with other randomized control studies corroborating this range of keratometry flattening [19-21]. While overall improvement in visual acuity has been modest and has generally not reached statistical significance, the failure rate (percentage of eyes with continued progression) has been relatively 
low at approximately $6-8 \%$ at 12 months postoperatively [22-24]. Additionally, the effects of treatment have been shown to remain stable for as long as 10 years in the original Dresden cohort [16].

\section{High-Fluence (Accelerated) Protocol}

Given the prolonged treatment time described by the Dresden protocol, many groups have attempted to achieve comparable outcomes with shorter treatment duration by altering irradiation fluence or riboflavin preparations, referred to as "accelerated CXL". In these protocols, high-energy application of UVA is utilized-up to $30 \mathrm{~mW} / \mathrm{cm}^{2}$, compared with the conventional $3 \mathrm{~mW} / \mathrm{cm}^{2}$-but the total energy limit is kept constant at $5.4 \mathrm{~J} / \mathrm{cm}^{2}$ to avoid phototoxicity [25]. A comparison between accelerated and conventional protocols showed no significant difference in safety, efficacy, or visual gains between the two groups, but the studies were constrained by a small number of cases and short follow-up time [26, 27]. While many other studies have since reported acceptable refractive and keratometric outcomes for these accelerated treatments, there is some concern that the biomechanical stiffening effects may not be as significant or long-lasting as with the conventional protocol, but this remains to be corroborated with long-term outcomes of randomized controlled trials $[28,29]$.

\section{Transepithelial CXL}

Another modification to the Dresden protocol that has arisen over the past decade is the attempt to limit disturbance of the corneal epithelium to hasten postoperative healing and further protect the endothelium (especially in cases of thinner corneas), nicknamed "epi-on CXL" [30]. This has necessitated modifications to the riboflavin solution or specialized procedures to increase corneal epithelial permeability in order to achieve adequate penetration of both the riboflavin cross-linking agent and UVA light. For example, chemical enhancers such as trometamol, benzalkonium chloride (BAK), ethylenediaminetetraacetic acid (EDTA), and gentamicin have been added concurrently with the riboflavin solution to loosen epithelial tight junctions; iontophoresis systems have attempted electrical disruption of the epithelium to promote intrastromal delivery of riboflavin; and superficial intrastromal administration of riboflavin via femtosecond laser-generated corneal pockets have also been reported [30-32]. However, these protocols remain controversial, as most have resulted in suboptimal results compared with conventional CXL, with a few recent small studies showing somewhat comparable but limited outcomes between the groups over a 2-year period [33]. These "epi-on" methods may continue to gain popularity as improved techniques are developed for enhancing intrastromal riboflavin diffusion to efficacy levels consistently comparable to conventional protocols.

\section{COMBINED CXL PROCEDURES FOR REFRACTIVE CORRECTION (CXL PLUS) (TABLE 1)}

\section{Combined CXL and Photorefractive Keratectomy (PRK)}

Topography-guided PRK was the first reported combined CXL treatment performed, initially in a keratoconus patient who underwent unilateral PRK 1 year after undergoing CXL, with excellent visual outcome (uncorrected visual acuity [UCVA] 20/20, BCVA 20/15) [34]. Based on the anecdotal success of a few such patients, it was postulated that simultaneous topography-guided PRK with CXL might be an alternative option for optimizing refractive outcomes of keratoconus with one treatment, and the Athens protocol was subsequently developed [35]. This procedure involves sequential excimer laser debridement of epithelium $(50 \mu \mathrm{m})$ and partial topographyguided excimer laser stromal ablation (maximum $80 \mu \mathrm{m})$, followed by high-fluence CXL $\left(10 \mathrm{~mW} / \mathrm{cm}^{2}\right.$ for $\left.10 \mathrm{~min}\right)[36,37]$. Significant fluctuations in refractive and topometric measures were noted within the first postoperative 


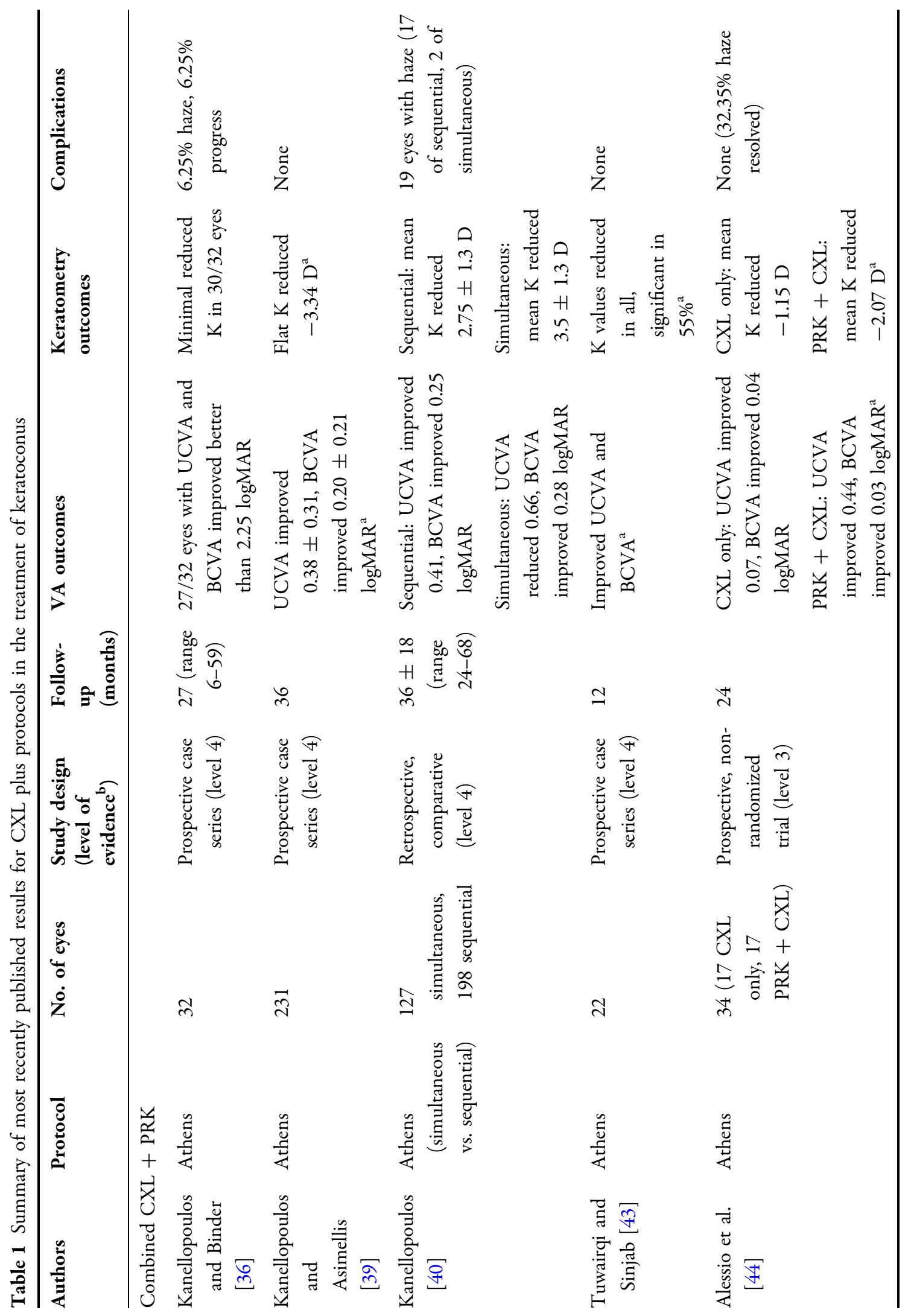




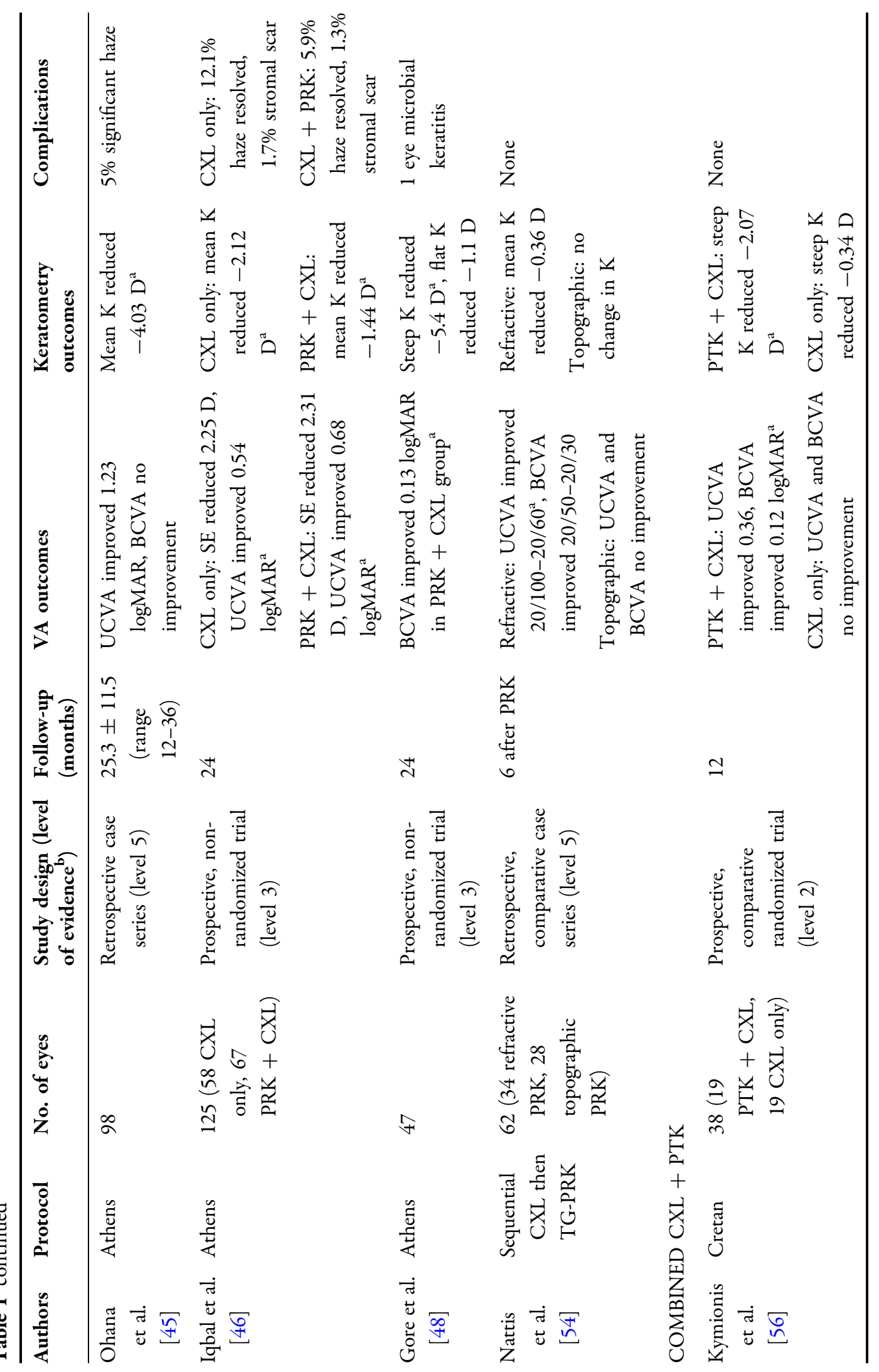




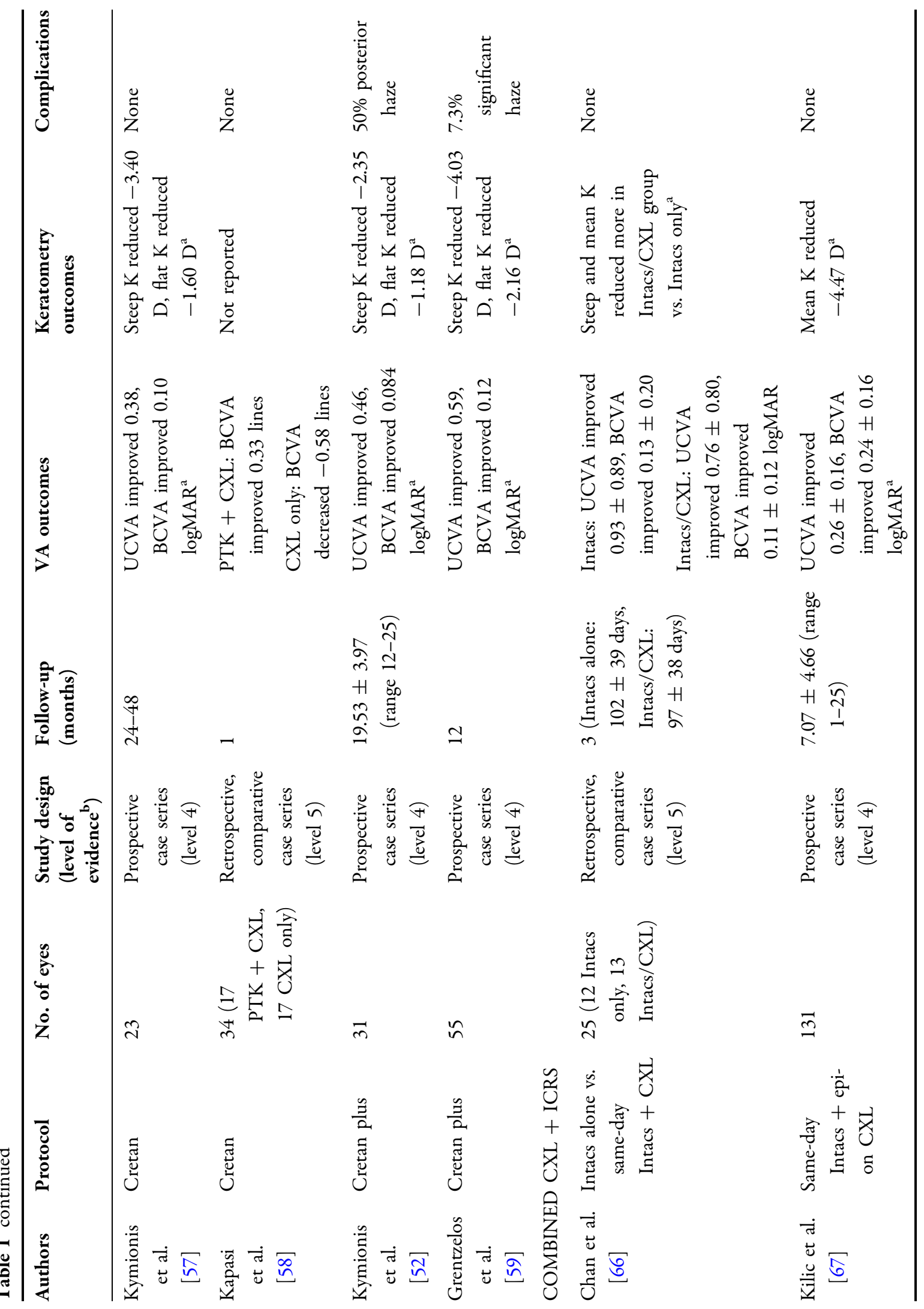




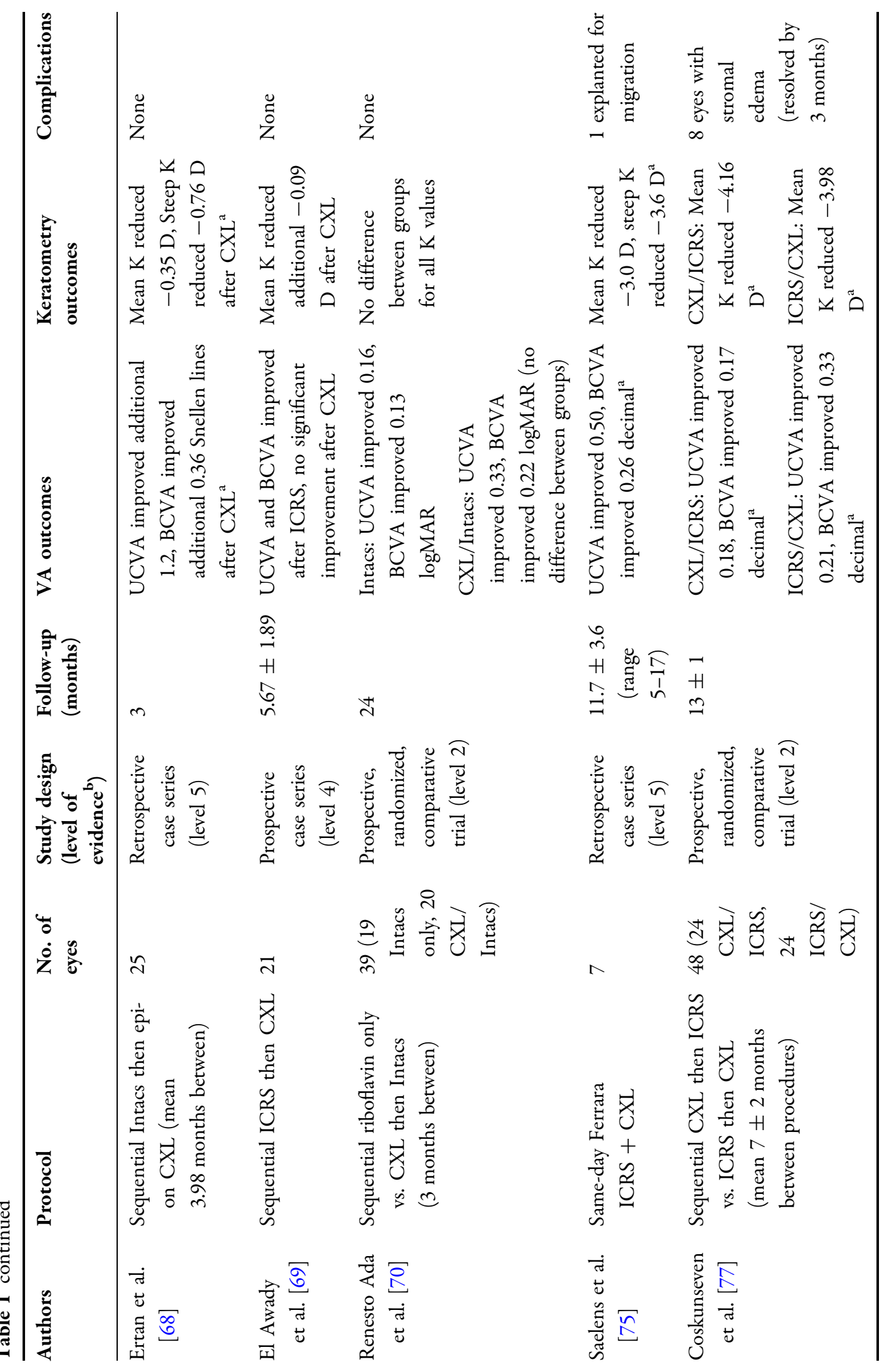




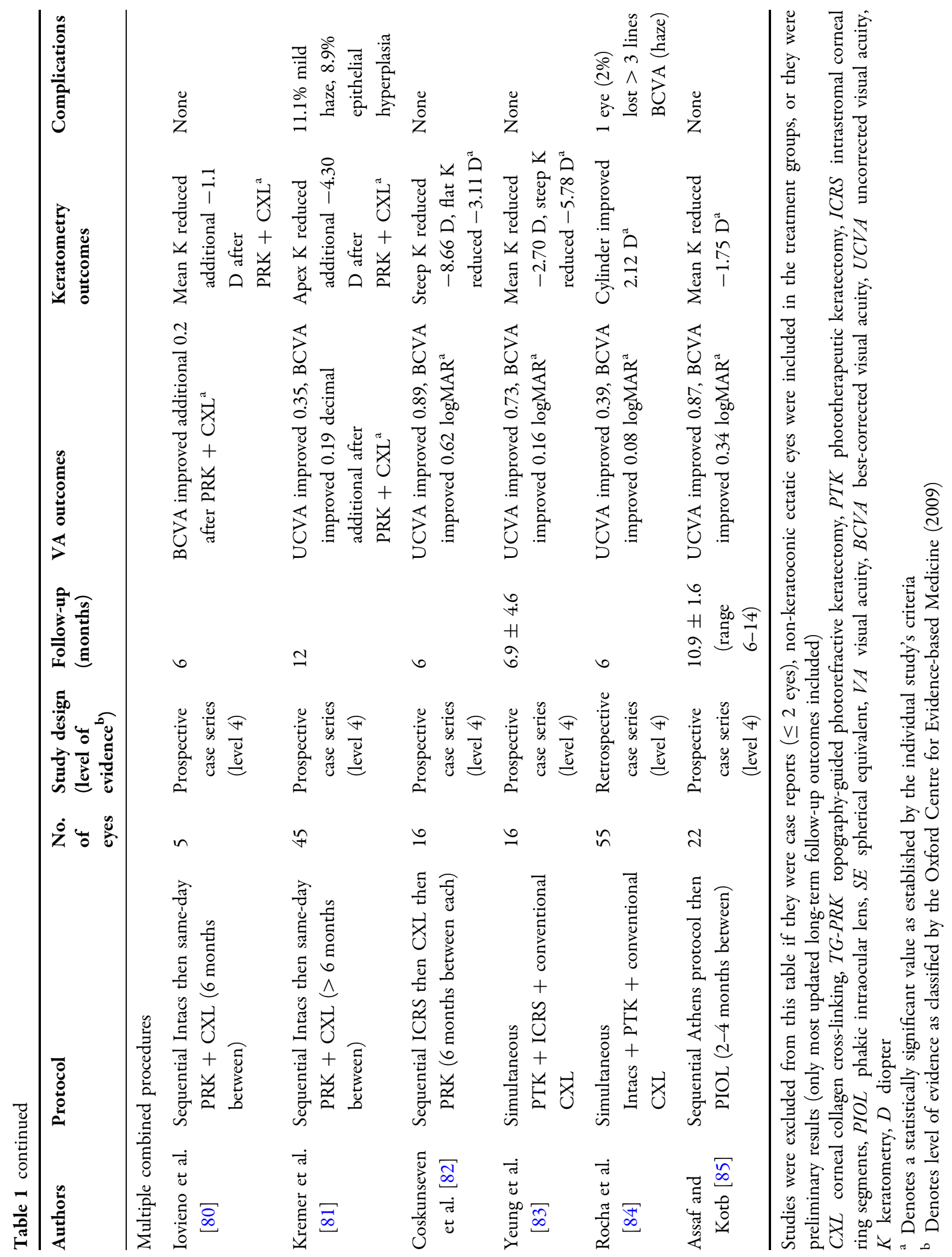


6 months, but 12-month postoperative results showed significant improvement in multiple topographic indices, independent of keratoconus grade or imaging platform (Scheimpflug vs. Placido disc), with only minimal changes in visual acuity from preoperative measures (gain $+0.19 \pm 0.20$ decimal BCVA at best) [38]. Further analysis of 231 eyes revealed gradual stabilization but persistent flattening of pachymetric and topographic indices over a 3-year period, implying a downstream effect of CXL-induced stromal changes to promote longer-term corneal flattening and thickening, and cautioning against overcorrection with this combined protocol [39]. Of note, while UCVA and BCVA improved postoperatively on average throughout the 3-year follow-up period, there was considerable variability in standard deviations equivalent to the absolute gains measured (range UCVA -0.34 to +1.10 , BCVA -0.32 to +0.90 ) [39]. Because these studies were primarily prospective observational trials without a control group, the true significance of the reported outcomes may be overstated.

Controversy remains around whether simultaneous procedures or sequential CXL followed by PRK is optimal. Kanellopoulos et al. initially showed same-day topography-guided PRK with CXL as more effective for visual rehabilitation (as incorporated into the Athens protocol), with subsequent studies corroborating increased efficacy of simultaneous CXL and PRK compared with CXL alone [40-45]. However, a recent prospective multicenter study comparing conventional CXL (Dresden protocol) to simultaneous combined PRK and CXL (Athens protocol) showed nearly equivalent results in visual outcomes and refractive improvement after 2 years, suggesting that there may not be a significant benefit with the Athens protocol over the longer-term refractive advantages of the conventional protocol [46]. Primary considerations for planning simultaneous combined CXL and PRK concern ablation depth and corneal thickness, so most protocols specify a maximum ablation depth between 50 and $80 \mu \mathrm{m}[34,41,42]$.

Another major concern with simultaneous procedures is in treatment planning, as CXL alone has been shown to gradually induce changes in anterior corneal curvature that do not stabilize until 6-12 months postoperatively [19]. It is therefore difficult to determine the precise ablation pattern or refraction that simultaneous topography-guided PRK should target, as the combination of procedures introduces significant unpredictability into current software models. A few groups have examined high-resolution wavefront-guided (HRWG) PRK or special treatment algorithms designed to target higher-order aberrations (HOAs) combined with accelerated CXL, with promising results from small preliminary studies demonstrating UCVA and BCVA gains without compromising CXL efficacy at 12-24 months postoperatively $[47,48]$. Another technique has utilized topography-guided PRK in conjunction with customized, variable-pattern CXL ("enhanced Athens protocol"), with preliminary data also showing encouraging improvements in UCVA, and keratometry values remaining stable, at 3-year follow-up [49]. However, these studies only enrolled patients with mild to moderate disease without severe ectasia, as demonstrated by relatively preserved preoperative corneal thickness and keratometry readings.

Additionally, increased risk of stromal haze after simultaneous combined procedures remains a substantial barrier to generalized adoption of this treatment method [50, 51]. Consequently, the use of mitomycin $\mathrm{C}$ following PRK during combined CXL plus treatments remains a topic of debate: while most protocols used mitomycin C $0.02 \%$ for $20-30 \mathrm{~s}$ after ablation, all reported postoperative haze formation. Kymionis et al. specifically avoided it due to the theoretical advantage that crosslinking itself leads to additional depopulation of anterior stromal keratocytes and may therefore reduce the risk of haze $[40,52]$. However, there was also mild (grade 1) posterior haze formation in $50 \%$ of their patients at 1 year, as is sometimes seen in patients undergoing CXL alone [52]. This unique pattern of haze formation suggests that the mechanism of haze induction is still poorly understood, and thus prevention remains challenging.

A recent meta-analysis revealed greater improvements in BCVA, spherical equivalent, 
astigmatism, and disease stability without progression in the sequential protocol studies, though direct comparisons between the studies were limited by the variety of different protocols used [53]. Since the ablation rate in crosslinked stroma may differ from that in native tissue, the refractive outcomes may be more unpredictable [53]. Early results from a retrospective US series of CXL patients undergoing sequential topography-guided PRK showed a significant improvement in UCVA and BCVA compared with those who received only CXL [54]. While these studies all intimate significant additive visual gains with combined PRK and $\mathrm{CXL}$, further investigation is necessary to delineate optimal treatment conditions for this combined procedure and to identify specific disease subgroups who are more likely to benefit.

\section{Combined CXL and Phototherapeutic Keratectomy (PTK)}

As an alternative to mechanical epithelial debridement, transepithelial PTK has been proposed to both remove epithelium and increase anterior stromal regularity by decreasing keratoconic astigmatism [55]. Named the Cretan protocol, this arose from a case report of a keratoconus patient who demonstrated visual and topographic improvement after PTK-based epithelial removal during conventional CXL [55]. The Cretan protocol includes transepithelial PTK ablation of $50 \mu \mathrm{m}$ intended depth in a 6.5-7.0 $\mathrm{mm}$ zone, then mechanical debridement to enlarge the de-epithelialized area to an 8.0-9.0 $\mathrm{mm}$ zone, followed by conventional CXL [56]. Subsequent case-control studies showed comparable safety, with improvements in visual and refractive outcomes, in a small series of 23 eyes followed for at least 24 months, with seven eyes followed through 4 years postoperatively [57]. Based on these reports, it is hypothesized that partial removal of Bowman's layer over the keratoconic corneal apex adds refractive advantage over CXL alone [58]. Unfortunately, reports on such small numbers of treated eyes preclude meaningful conclusions on the overall management of keratoconus patients.

The Cretan protocol has been further extended to include adjunctive PRK and for supplemental refractive correction. Referred to as the Cretan plus protocol, transepithelial PTK is combined with conventional PRK (maximum $50 \mu \mathrm{m}$ ablation depth) and simultaneous conventional CXL [59]. This small, uncontrolled case series showed significant improvements in UCVA, BCVA, keratometry, and refractive values, with acceptable safety metrics, at 1 year. A similar protocol allowing $75 \mu \mathrm{m}$ ablation depth by PRK also showed promising early results in a small cohort of Chinese patients [60]. This particular combination may therefore be worth further exploring to determine whether there is an added benefit to laser-assisted de-epithelialization rather than mechanical debridement along with combined PRK photoablation and CXL.

\section{Combined CXL and Conductive Keratoplasty (CK)}

Conductive keratoplasty (CK) is a noninvasive, tissue-preserving procedure that utilizes radiofrequency energy $(350 \mathrm{~Hz})$, which penetrates $90 \%$ of corneal stroma when applied directly to cause permanent collagen shrinkage [61]. It is traditionally approved for the treatment of low to moderate hyperopia and astigmatism if applied circumferentially around the corneal mid-periphery to cause central steepening, but a few attempts have been made to apply it to keratoconic corneas to achieve selective steepening in flat, non-ectatic areas. Kato et al. first evaluated "topography-guided conductive keratoplasty" (TGCK) for moderate to advanced keratoconus by delivering radioprobe applications based on preoperative topography and intraoperative keratometry in 21 eyes; while small, non-statistically significant improvements in UCVA and BCVA were noted at 1 year postoperatively in the 10 eyes completing follow-up, regression toward baseline keratometry values was noted, similar to those observed with classic CK treatments in hyperopic corneas [61]. Initial endeavors to combine 
CK with simultaneous conventional CXL in order to theoretically stabilize CK effects further showed significant regression of CK effects by postoperative month 3 [62]. More recently, Rubinfeld et al. reported two cases of post-LASIK ectasia that failed conventional CXL and were subsequently treated with TGCK, aiming for immediate 4.0-6.0 D overcorrection, followed by sequential transepithelial CXL with a proprietary riboflavin solution after $24 \mathrm{~h}$, which resulted in stable improvements in BCVA, astigmatism, and keratometric flattening at 1-3 years postoperatively [63, 64]. While these results may not be generalizable in the long term to the keratoconus population, and the safety profile remains unknown, these case reports of early successes with this new combined protocol are encouraging.

\section{Combined CXL and Intrastromal Corneal Ring Segments (ICRS)}

Originally developed as a treatment for myopia, intrastromal corneal ring segments (ICRS) are polymethyl methacrylate (PMMA) pieces implanted in the mid-peripheral deep stroma to reduce central corneal curvature. Intacs ${ }^{\circledR}$ (Addition technology, Inc.) and Ferrara Rings (Mediphacos, Inc.) are the most common commercially available brands. While ICRS have been shown to be effective in improving visual acuity in keratoconus by regularizing and decreasing pathologic corneal steepening and irregular astigmatism, their use alone does not prevent disease progression [65]. They have recently regained popularity as an adjunctive treatment with CXL for mild to moderate keratoconus, with studies showing small improvements in UCVA associated with significant reductions in $\mathrm{K}$ values, suggesting normalization of corneal shape, albeit with variable improvement over ICRS implantation alone [66-70]. Ultimate surgical success is highly dependent upon patient selection and various intraoperative factors, including proper ring placement, accurate depth of implantation, and optical zone diameter. Ring extrusion remains a potential postoperative complication, with rates up to $10 \%$ reported in ICRS implantation alone in keratoconus patients, though only a few rare cases of postoperative migration have been reported thus far during the short $(<1$ year) follow-up for the combined CXL and ICRS procedures [71-75]. The feasibility of ICRS combined with CXL is also currently limited by moderate predictability of refractive outcomes, and the overall costs of the procedure may be dramatically increased.

Moreover, and similar to other combined protocols, the optimal timing for ICRS implantation remains unclear: simultaneous ICRS with CXL, CXL followed by ICRS, or ICRS followed by CXL. A few studies have shown that CXL followed by ICRS has the least postoperative improvement, with increased difficulty of surgical dissection, femtosecond laser energy needs, and risk of corneal haze that may be related to channel generation in already crosslinked corneal tissue [70, 76, 77]. It is also hypothesized that there may be an additive effect of same-day combined ICRS and CXL, with CXL serving as a stabilizing procedure, enhancing the benefits of ICRS $[66,67,72,73,78]$. Interestingly, reports of purposefully delayed ICRS explantation (not for extrusion) after initial combined same-day CXL described some persistence in beneficial topographic changes without complete regression to pre-CXL/ICRS values, implying that CXL may prolong the structural effects of ICRS despite the reversibility of implants [79]. However, not enough large studies with extended follow-up have been conducted to fully determine the ideal technical protocol and long-term outcomes for combined ICRS with CXL.

\section{Multiple Combined CXL Procedures}

It appears that even combining CXL with other refractive procedures does not currently lead to optimal visual acuity, so some studies have addressed potential combinations of multiple procedures with CXL. Triple therapy with CXL, PRK, and ICRS and with PTK, CXL, and ICRS has been reported in a few small case series as both a simultaneous and staged procedure. Improvements in vision, topography, and refraction (including reduced total aberrations) were 
noted after 6-12 months of follow-up regardless of whether the procedures were performed simultaneously or sequentially with ICRS implantation followed by combined CXL-PRK [80-84]. Another triple therapy with simultaneous CXL and PRK (Athens protocol) followed by delayed phakic intraocular lens (PIOL) implantation after 2-4 months has been described as well, with short-term (6-month) follow-up results in a small case series showing significant stabilization of uncorrected vision gains [85]. These triple procedures also warrant further large-scale studies to elucidate whether their overall safety profile is acceptable and whether there is enough supplementary benefit to justify the additional risk.

\section{GRADE PRACTICE RECOMMENDATIONS}

In general, no level 1 evidence yet exists for any of these combined CXL plus procedures, as no large randomized controlled trials have been conducted or published results. Most studies have also been relatively small case series, and findings have varied among different protocols and groups. Thus, none merit a grade A or B recommendation at this time.

For combined CXL with PRK (Athens protocol), there is consistent level 4 evidence that shows its safety and efficacy. However, there is some controversy within level 3 studies that suggest the longer-term results may not be significantly improved compared with CXL alone $[44,46]$. For this reason, we offer a grade C recommendation for this procedure.

For combined CXL with ICRS, we also offer a grade $\mathrm{C}$ recommendation, due to consistent level 4 evidence showing acceptable safety and efficacy outcomes. There are a few level 2 studies that show questionable benefit of the combined procedure over ICRS alone, but all were small, relatively underpowered studies.

For all other combined CXL plus procedures (CXL with PTK, CK, or multiple combined procedures), we can only offer a grade $\mathrm{D}$ recommendation at this time, due to the paucity of higher-level evidence showing a significant benefit with the combined protocols.

\section{FUTURE OF REFRACTIVE SURGICAL OPTIONS FOR KERATOCONUS}

Despite decades of research, the complete pathophysiology of keratoconus remains enigmatic, although detection methods have advanced enough that more keratoconus patients may be diagnosed early in the disease process, when visual acuity is relatively preserved. CXL in these early stages will hopefully prevent these patients from having to undergo additional procedures to address their refractive consequences. However, the management of patients with moderate to advanced disease remains challenging; although the plethora of methods reviewed above provide greater flexibility to both patients and clinicians, representing a significant expansion of treatment options compared with previous decades, there is still work to be done until the elusive "cure" can be attained. Since CXL alone does not fully address the refractive visual component of keratoconus therapy, many of the described combined CXL plus protocols require further investigation with larger, controlled trials with longer follow-up periods to better optimize outcomes. As topography-driven refractive technologies continue to progress, there is great hope that these protocols may be able to be personalized for each individual patient and their particular disease status.

Furthermore, CXL alone may be able to be developed to achieve a larger standalone impact on refractive error. Efforts to generate customized cross-linking systems may produce algorithms by which CXL treatments can be topography-guided [86]. Even prior to using CXL to halt progression of established keratoconus, there has been some recent exploration of utilizing CXL as a prophylactic measure in conjunction with LASIK or PRK on healthy myopes, suggesting that simultaneous CXL may even reduce postoperative regression along with development of ectasia $[87,88]$. With these endeavors to actively prevent corneal ectatic disorders from becoming clinically manifest, it is imaginable that CXL may eventually be able to supplant entirely or remain a fundamental 
adjunct in our armamentarium of refractive surgical procedures.

\section{ACKNOWLEDGEMENTS}

This supplement was not sponsored by outside commercial interests.

Funding. No funding or sponsorship was received for this study or publication of this article.

Authorship. All named authors meet the International Committee of Medical Journal Editors (ICMJE) criteria for authorship for this article, take responsibility for the integrity of the work as a whole, and have given their approval for this version to be published.

Disclosures. Angela Y. Zhu has a new affiliation with Bascom Palmer Eye Institute, University of Miami Miller School of Medicine, Miami, FL, and has nothing to declare. Albert S. Jun and Uri S. Soiberman have nothing to declare.

Compliance with Ethics Guidelines. This article is based on previously conducted studies and does not contain any studies with human participants or animals performed by any of the authors.

Open Access. This article is distributed under the terms of the Creative Commons Attribution-NonCommercial 4.0 International License (http://creativecommons.org/licenses/ by-nc/4.0/), which permits any noncommercial use, distribution, and reproduction in any medium, provided you give appropriate credit to the original author(s) and the source, provide a link to the Creative Commons license, and indicate if changes were made.

\section{REFERENCES}

1. Spörl E, Huhle M, Kasper M, Seiler T. Increased rigidity of the cornea caused by intrastromal crosslinking. Ophthalmologe. 1997;94(12):902-6.
2. Shetty R, Kaweri L, Pahuja N, Nagaraja H, Wadia K, Jayadev C, Nuijts R, Arora V. Current review and a simplified "five-point management algorithm" for keratoconus. Indian J Ophthalmol. 2015;63(1):46-53.

3. Rabinowitz YS. Keratoconus. Surv Ophthalmol. 1998;42(4):297-319.

4. Mohammadpour M, Heidari Z, Hashemi H. Updates on Management for Keratoconus. J Curr Ophthalmol. 2017;30(2):110-24.

5. Klintworth GK, Damms T. Corneal dystrophies and keratoconus. Curr Opin Ophthalmol. 1995;6(4):44-56.

6. Godefrooij DA, de Wit GA, Uiterwaal CS, Imhof SM, Wisse RP. Age-specific incidence and prevalence of keratoconus: a nationwide registration study. Am J Ophthalmol. 2017;175:169-72.

7. Mathews PM, Lindsley K, Aldave AJ, et al. Etiology of global corneal blindness and current practices of corneal transplantation: a focused review. Cornea. 2018;37(9):1198-203.

8. Davidson AE, Hayes S, Hardcastle AJ, Tuft SJ. The pathogenesis of keratoconus. Eye. 2014;28:189-95.

9. Chaerkady R, Shao H, Scott SG, Pandey A, Jun AS, Chakravarti $S$. The keratoconus corneal proteome: loss of epithelial integrity and stromal degeneration. J Proteomics. 2013;11(87):122-31.

10. Kim WJ, Rabinowitz YS, Meisler DM, Wilson SE. Keratocyte apoptosis associated with keratoconus. Exp Eye Res. 1999;69:475-81.

11. Joseph R, Srivastava OP, Pfister RR. Differential epithelial and stromal protein profiles in keratoconus and normal human corneas. Exp Eye Res. 2011;92:282-98.

12. Ghosh A, Zhou L, Gosh A, Shetty R, Beureman R. Proteomic and gene expression patterns in keratoconus. Indian J Ophthalmol. 2013;61:389-91.

13. Wollensak G, Spoerl E, Seiler T. Riboflavin/ultraviolet-A-induced collagen crosslinking for the treatment of keratoconus. Am J Ophthalmol. 2003;135(5):620-7.

14. Kymionis GD, Grentzelos MA, Liakopoulos DA, Paraskevopoulos TA, Klados NE, Tsoulnaras KI, Kankariya VP, Pallikaris IG. Long-term follow-up of corneal collagen cross-linking for keratoconus-the Cretan study. Cornea. 2014;33(10):1071-9.

15. O'Brart DP, Patel P, Lascaratos G, Wagh VK, Tam C, Lee J, O'Brart NA. Corneal cross-linking to halt the progression of keratoconus and corneal ectasia: 
seven-year follow-up. Am J Ophthalmol. 2015;160(6):1154-63.

16. Raiskup F, Theuring A, Pillunat LE, Spoerl E. Corneal collagen crosslinking with riboflavin and ultraviolet-A light in progressive keratoconus: tenyear results. J Cataract Refract Surg. 2015;41(1):41-6.

17. Spoerl E, Mrochen M, Sliney D, Trokel S, Seiler T. Safety of UVA-riboflavin cross-linking of the cornea. Cornea. 2007;26(4):385-9.

18. Baiocchi S, Mazzotta C, Cerretani D, Caporossi T, Caporossi A. Corneal crosslinking: riboflavin concentration in corneal stroma exposed with and without epithelium. J Cataract Refract Surg. 2009;35:893-9.

19. Hersh PS, Stulting RD, Muller D, Durrie DS, Rajpal RK. United States Crosslinking Study Group United States. Multicenter Clinical Trial of Corneal Collagen Crosslinking for Keratoconus Treatment. Ophthalmology. 2017;124(9):1259-70.

20. Caporossi A, Mazzotta C, Baiocchi S, Caporossi T. Long-term results of riboflavin ultraviolet A corneal collagen cross-linking for keratoconus in Italy: the Siena Eye Cross Study. Am J Ophthalmol. 2010;149:585-93.

21. Hersh PS, Greenstein SA, Fry KL. Corneal collagen crosslinking for keratoconus and corneal ectasia: one-year results. J Cataract Refract Surg. 2011;37:149-60.

22. O'Brart DP, Chan E, Samaras K, Patel P, Shah SP. A randomised, prospective study to investigate the efficacy of riboflavin/ultraviolet A $(370 \mathrm{~nm})$ corneal collagen cross-linkage to halt the progression of keratoconus. Br J Ophthalmol. 2011;95:1519-24.

23. Wittig-Silva C, Chan E, Islam FMA, Wu T, Whiting M, Snibson GR. A randomized, controlled trial of corneal collagen cross-linking in progressive keratoconus: three-year results. Ophthalmology. 2014;121:812-21.

24. Koller T, Mrochen M, Seiler T. Complication and failure rates after corneal crosslinking. J Cataract Refract Surg. 2009;35(8):1358-62.

25. Elbaz U, Shen C, Lichtinger A, et al. Accelerated (9$\mathrm{mW} / \mathrm{cm}^{2}$ ) corneal collagen crosslinking for keratoconus-A 1-year follow-up. Cornea. 2014;33(8):769-73.

26. Cınar Y, Cingü AK, Türkcü FM, Çınar T, Yüksel H, Özkurt ZG, Çaça I. Comparison of accelerated and conventional corneal collagen cross-linking for progressive keratoconus. Cutan Ocul Toxicol. 2014;33:218-22.
27. Kanellopoulos AJ. Long term results of a prospective randomized bilateral eye comparison trial of higher fluence, shorter duration ultraviolet A radiation, and riboflavin collagen cross linking for progressive keratoconus. Clin Ophthalmol. 2012;6:97-101.

28. Waszczykowska A, Jurowski P. Two-year accelerated corneal crosslinking outcome in patients with progressive keratoconus. Biomed Res Int. 2015;2015:325157.

29. Shetty R, Pahuja NK, Nuijts RM, Ajani A, Jayadev C, Sharma C, Nagaraja H. Current protocols of corneal collagen cross-linking: visual, refractive, and tomographic outcomes. Am J Ophthalmol. 2015;160(2):243-9.

30. Raiskup F, Spoerl E. Corneal crosslinking with riboflavin and ultraviolet A. I. Principles. Ocul Surf. 2013;11:65-74.

31. Mastropasqua L, Nubile M, Calienno R, Mattei PA, Pedrotti E, Salgari N, Mastropasqua R, Lanzini M. Corneal cross-linking: intrastromal riboflavin concentration in iontophoresis-assisted imbibition versus traditional and transepithelial techniques. Am J Ophthalmol. 2014;157:623-30.

32. Kanellopoulos AJ. Collagen cross-linking in early keratoconus with riboflavin in a femtosecond lasercreated pocket: initial clinical results. J Refract Surg. 2009;25:1034-7.

33. Cifariello F, Minicucci M, Di Renzo F, Di Taranto D, Coclite G, Zaccaria S, De Turris S, Costagliola C. Epioff versus epi-on corneal collagen cross-linking in keratoconus patients: a comparative study through 2-year follow-up. J Ophthalmol. 2018;2018:4947983.

34. Kanellopoulos AJ, Binder PS. Collagen cross-linking (CCL) with sequential topography-guided PRK: a temporizing alternative for keratoconus to penetrating keratoplasty. Cornea. 2007;26:891-5.

35. Krueger RR, Kanellopoulos AJ. Stability of simultaneous topography-guided photorefractive keratectomy and riboflavin/UVA cross-linking for progressive keratoconus: case reports. J Refract Surg. 2010;26(10):S827-32.

36. Kanellopoulos AJ, Binder PS. Management of corneal ectasia after LASIK with combined, same-day, topography-guided partial transepithelial PRK and collagen cross-linking: the Athens protocol. J Refract Surg. 2011;27:323-31.

37. Kanellopoulos AJ, Asimellis G. Corneal refractive power and symmetry changes following normalization of ectasias treated with partial topographyguided PTK combined with higher-fluence CXL 
(The Athens Protocol). J Refract Surg. 2014;30(5):342-6.

38. Kanellopoulos AJ, Asimellis G. Comparison of Placido disc and Scheimpflug image-derived topography-guided excimer laser surface normalization combined with higher fluence CXL: the Athens Protocol, in progressive keratoconus. Clin Ophthalmol. 2013;7:1385-96.

39. Kanellopoulos AJ, Asimellis G. Keratoconus management: long-term stability of topography-guided normalization combined with high-fluence CXL stabilization (The Athens Protocol). J Refract Surg. 2014;30(2):88-92.

40. Kanellopoulos AJ. Comparison of sequential vs same-day simultaneous collagen cross-linking and topography-guided PRK for treatment of keratoconus. J Refract Surg. 2009;25:S812-8.

41. Stojanovic A, Zhang J, Chen X, Nitter TA, Chen S, Wang Q. Topography-guided transepithelial surface ablation followed by corneal collagen cross-linking performed in a single combined procedure for the treatment of keratoconus and pellucid marginal degeneration. J Refract Surg. 2010;26:145-52.

42. Lin DT, Holland S, Tan JC, Moloney G. Clinical results of topography-based customized ablations in highly aberrated eyes and keratoconus/ectasia with cross-linking. J Refract Surg. 2012;28:S841-8.

43. Tuwairqi WS, Sinjab MM. Safety and efficacy of simultaneous corneal collagen cross-linking with topography-guided PRK in managing low-grade keratoconus: 1-year follow-up. J Refract Surg. 2012;28:341-5.

44. Alessio G, L'Abbate M, Sborgia C, La Tegola MG. Photorefractive keratectomy followed by crosslinking versus cross-linking alone for management of progressive keratoconus: two-year follow-up. Am J Ophthalmol. 2013;155:54-65.

45. Ohana O, Kaiserman I, Domniz Y, Cohen E, Franco O, Sela T, Munzer G, Varssano D. Outcomes of simultaneous photorefractive keratectomy and collagen crosslinking. Can J Ophthalmol. 2018;53(5):523-8.

46. Iqbal M, Elmassry A, Tawfik A, Elgharieb M, Nagy K, Soliman A, Saad H, Tawfik T, Ali O, Gad A, El Saman I, Radwan A, Elzembely H, Abou Ali A, Fawzy O. Standard cross-linking versus photorefractive keratectomy combined with accelerated cross-linking for keratoconus management: a comparative study. Acta Ophthalmol. 2019;97(4):e623-31.

47. Tamayo GE, Castell C, Vargas P, Polania E, Tamayo J. High-resolution wavefront-guided surface ablation with corneal cross-linking in ectatic corneas: a pilot study. Clin Ophthalmol. 2017;11:1777-83.

48. Gore DM, Leucci MT, Anand V, Fernandez-Vega Cueto L, Arba Mosquera S, Allan BD. Combined wavefront-guided transepithelial photorefractive keratectomy and corneal crosslinking for visual rehabilitation in moderate keratoconus. J Cataract Refract Surg. 2018;44:571-80.

49. Kanellopoulos AJ. Management of progressive keratoconus with partial topography-guided PRK combined with refractive, customized CXL-a novel technique: the enhanced Athens protocol. Clin Ophthalmol. 2019;13:581-8.

50. Kymionis GD, Portaliou DM, Diakonis VF, Kontadakis GA, Krasia MS, Papadiamantis AG, Coskunseven E, Pallikaris AI. Posterior linear stromal haze formation after simultaneous photorefractive keratectomy followed by corneal collagen crosslinking. Invest Ophthalmol Vis Sci. 2010;51:5030-3.

51. Moraes RLB, Ghanem RC, Ghanem VC, Santhiago MR. Haze and visual acuity loss after sequential photorefractive keratectomy and corneal crosslinking for keratoconus. J Refract Surg. 2019;35(2):109-14.

52. Kymionis GD, Portaliou DM, Kounis GA, Limnopoulou AN, Kontadakis GA, Grentzelos MA. Simultaneous topography-guided photorefractive keratectomy followed by corneal collagen crosslinking for keratoconus. Am J Ophthalmol. 2011;152:748-55.

53. Bardan AS, Lee $H$, Nanavaty MA. Outcomes of simultaneous and sequential cross-linking with excimer laser surface ablation in keratoconus. J Refract Surg. 2018;34(10):690-6.

54. Nattis A, Donnenfeld ED, Rosenberg E, Perry HD. Visual and keratometric outcomes of keratoconus patients after sequential corneal crosslinking and topography-guided surface ablation: early United States experience. J Cataract Refract Surg. 2018;44:1003-11.

55. Kymionis GD, Grentzelos MA, Karavitaki AE, Kounis GA, Kontadakis GA, Yoo S, Pallikaris IG. Transepithelial phototherapeutic keratectomy using a 213-nm solid-state laser system followed by corneal collagen crosslinking with riboflavin and UVA irradiation. J Ophthalmol. 2010;2010:146543.

56. Kymionis GD, Grentzelos MA, Kounis GA, Diakonis VF, Limnopoulou AN, Panagopoulou SI. Combined transepithelial phototherapeutic keratectomy and corneal collagen cross-linking for progressive keratoconus. Ophthalmology. 2012;119:1777-84. 
57. Kymionis GD, Grentzelos MA, Kankariya VP, Liakopoulos DA, Karavitaki AE, Portaliou DM, Tsoulnaras KI, Pallikaris IG. Long-term results of combined transepithelial phototherapeutic keratectomy and corneal collagen crosslinking for keratoconus: Cretan protocol. J Cataract Refract Surg. 2014;40:1439-45.

58. Kapasi M, Baath J, Mintsioulis G, Jackson WB, Baig K. Phototherapeutic keratectomy versus mechanical epithelial removal followed by corneal collagen crosslinking for keratoconus. Can J Ophthalmol. 2012;47:344-7.

59. Grentzelos MA, Kounis GA, Diakonis VF, Siganos CS, Tsilimbaris MK, Pallikaris IG, Kymionis GD. Combined transepithelial phototherapeutic keratectomy and conventional photorefractive keratectomy followed simultaneously by corneal crosslinking for keratoconus: Cretan protocol plus. J Cataract Refract Surg. 2017;43:1257-62.

60. Zhu W, Han Y, Cui C, Xu W, Wang X, Dou X, Xu L, $\mathrm{Xu} \mathrm{Y,} \mathrm{Mu} \mathrm{G}$. Corneal collagen crosslinking combined with phototherapeutic keratectomy and photorefractive keratectomy for corneal ectasia after laser in situ keratomileusis. Ophthalmic Res. 2018;59:135-41.

61. Kato N, Toda I, Kawakita T, Sakai C, Tsubota K. Topography-guided conductive keratoplasty: treatment for advanced keratoconus. Am J Ophthalmol. 2010;150(4):481-489.e1.

62. Kymionis GD, Kontadakis GA, Naoumidi TL, Kazakos DC, Giapitzakis I, Pallikaris IG. Conductive keratoplasty followed by collagen cross-linking with riboflavin-UV-A in patients with keratoconus. Cornea. 2010;29(2):239-43.

63. Rubinfeld RS, Epstein RH, Majmudar PA, Kim D, Choi M, Epstein RJ. Transepithelial crosslinking retreatment of progressive corneal ectasia unresponsive to classic crosslinking. J Cataract Refract Surg. 2017;43(1):131-5.

64. Mannschreck DB, Rubinfeld RS, Soiberman US, Jun AS. Diffuse lamellar keratitis after epi-off corneal crosslinking: an under-recognized complication? Am J Ophthalmol Case Rep. 2019;13:140-2.

65. Ganesh S, Shetty R, D'Souza S, Ramachandran S, Kurian M. Intrastromal corneal ring segments for management of keratoconus. Indian J Ophthalmol. 2013;61(8):451-5.

66. Chan CCK, Sharma M, Wachler BS. Effect of inferior-segment Intacs with and without C3-R on keratoconus. J Cataract Refract Surg. 2007;33:75-80.

67. Kilic A, Kamburoglu G, Akinci A. Riboflavin injection into the corneal channel for combined collagen crosslinking and intra-stromal corneal ring segment implantation. J Cataract Refract Surg. 2012;38:878-83.

68. Ertan Q, Karacal H, Kamburoglu G. Refractive and topographic results of transepithelial cross-linking treatment in eyes with Intacs. Cornea. 2009;28:719-23.

69. El Awady H, Shawky M, Ghanem AA. Evaluation of collagen crosslinking in keratoconus eyes with Kera intra-corneal ring implantation. Eur J Ophthalmol. 2012;22:62-8.

70. Renesto Ada C, Melo LA Jr, Sartori Mde F, Campos M. Sequential topical riboflavin with or without ultraviolet a radiation with delayed intracorneal ring segment insertion for keratoconus. Am J Ophthalmol. 2012;153:982-93.

71. Zare MA, Hashemi H, Salari MR. Intracorneal ring segment implantation for the management of keratoconus: safety and efficacy. J Cataract Refract Surg. 2007;33(11):1886-91.

72. Yeung SN, Ku JY, Lichtinger A, Low SA, Kim P, Rootman DS. Efficacy of single or paired intrastromal corneal ring segment implantation combined with collagen crosslinking in keratoconus. J Cataract Refract Surg. 2013;39(8):1146-51.

73. Gordillo $\mathrm{CH}$, Grandin JC, Zaldivar R, Zaldivar R, Lotfi AC. Efficacy of intrastromal corneal ring segments combined with flash collagen cross-linking in keratoconus. Cornea. 2017;36(2):144-7.

74. Ayar O, Muftuoglu O, Akdemir MO, Ozmen MC. Results of ethanol-assisted epithelium-on corneal cross-linking with and without intrastromal corneal ring implantation. Int Ophthalmol. 2019;39(3):651-9.

75. Saelens IE, Bartels MC, Bleyen I, Van Rij G. Refractive, topographic, and visual outcomes of same-day corneal cross-linking with Ferrara intracorneal ring segments in patients with progressive keratoconus. Cornea. 2011;30:1406-8.

76. El-Raggal TM. Effect of corneal collagen crosslinking on femtosecond laser channel creation for intrastromal corneal ring segment implantation in keratoconus. J Cataract Refract Surg. 2011;37:701-5.

77. Coskunseven E, Jankov M, Hafezi F, Atun S, Arslan E, Kymionis GD. Effect of treatment sequence in combined intrastromal corneal rings and corneal collagen cross-linking for keratoconus. J Cataract Refract Surg. 2009;35:2084-91.

78. Hashemi H, Alvani A, Seyedian MA, Yaseri M, Khabazkhoob M, Esfandiari H. Appropriate 
sequence of combined intracorneal ring implantation and corneal collagen cross-linking in keratoconus: a systematic review and meta-analysis. Cornea. 2018;37(12):1601-7.

79. Yeung SN, Lichtinger A, Ku JY, Kim P, Low SA, Rootman DS. Intracorneal ring segment explantation after intracorneal ring segment implantation combined with same-day corneal collagen crosslinking in keratoconus. Cornea. 2013;32(12):1617-20.

80. Iovieno A, Légaré ME, Rootman DB, Yeung SN, Kim $\mathrm{P}$, Rootman DS. Intracorneal ring segments implantation followed by same-day photorefractive keratectomy and corneal collagen cross-linking in keratoconus. J Refract Surg. 2011;27:915-8.

81. Kremer I, Aizenman I, Lichter H, Shayer S, Levinger S. Simultaneous wavefront-guided photorefractive keratectomy and corneal collagen crosslinking after intrastromal corneal ring segment implantation for keratoconus. J Cataract Refract Surg. 2012;38:1802-7.

82. Coskunseven E, Jankov MR II, Grentzelos MA, Plaka AD, Limnopoulou AN, Kymionis GD. Topographyguided transepithelial PRK after intracorneal ring segments implantation and corneal collagen CXL in a three-step procedure for keratoconus. J Refract Surg. 2013;29:54-8.

83. Yeung SN, Low SA, Ku JY, et al. Transepithelial phototherapeutic keratectomy combined with implantation of a single inferior intrastromal corneal ring segment and collagen crosslinking in keratoconus. J Cataract Refract Surg. 2013;39:1152-6.

84. Rocha G, Ibrahim T, Gulliver E, Lewis K. Combined phototherapeutic keratectomy, intracorneal ring segment implantation, and corneal collagen crosslinking in keratoconus management. Cornea. 2019;38(10):1233-8.

85. Assaf A, Kotb A. Simultaneous corneal crosslinking and surface ablation combined with phakic intraocular lens implantation for managing keratoconus. Int Ophthalmol. 2015;35:411-9.

86. Belin MW, Lim L, Rajpal RK, Hafezi F, Gomes JAP, Cochener B. Corneal cross-linking: current USA status: report from the cornea society. Cornea. 2018;37(10):1218-25.

87. Chan TCY, Ng ALK, Chan KKW, Cheng GPM, Wong IYH, Jhanji V. Combined application of prophylactic corneal cross-linking and laser in situ keratomileusis-a review of literature. Acta Ophthalmol. 2017;95:660-4.

88. Lee H, Roberts CJ, Ambrósio R Jr, Elsheikh A, Kang DSY, Kim TI. Effect of accelerated corneal crosslinking combined with transepithelial photorefractive keratectomy on dynamic corneal response parameters and biomechanically corrected intraocular pressure measured with a dynamic Scheimpflug analyzer in healthy myopic patients. J Cataract Refract Surg. 2017;43(7):937-45. 